\title{
Comparison of the oxygen sensor performance of some pyrene derivatives in silicone polymer matrix
}

\author{
Bharathibai J. Basu*, K.S. Rajam \\ Surface Engineering Division, National Aerospace Laboratories, Bangalore 560017, India \\ Received 22 October 2003; received in revised form 16 December 2003; accepted 17 December 2003
}

\begin{abstract}
Pyrene and its derivatives find application as fluorescent probes for monitoring the oxygen concentrations in air and various fluids. Photophysical characteristics of some commercially available derivatives of pyrene in silicone polymer matrix were studied in order to find out whether they could be employed as a substitute for pyrene in the pressure sensitive paint (PSP). The derivatives under study were: 1-pyrenyl methanol (PMO), 1-pyrenyl butanol (PBO), 1-pyrene butyric acid (PBA), and 1-pyrene acetic acid (PAA). Distinct differences were observed in the fluorescence emission spectra of the silicone coatings of derivatives depending on the functional group attached to pyrene. The oxygen quenching sensitivity (OQS) of these probes were evaluated and compared to that of pyrene. It was found that the fluorescence spectra of all silicone coatings of pyrene derivatives exhibited monomer and excimer peaks. The excimer emission of PBO in silicone matrix was oxygen sensitive whereas the coatings of the other three pyrene derivatives exhibited poor oxygen quenching.

(C) 2003 Elsevier B.V. All rights reserved.
\end{abstract}

Keywords: Pyrene derivative; Fluorescence quenching; Pressure sensitive paint; Oxygen sensor

\section{Introduction}

Pyrene and some its derivatives are often used as fluorescent probes for oxygen sensing applications because they have a long excited state lifetime and are efficiently quenched by oxygen [1-6]. The optical oxygen sensor is based on the quenching of luminescence by oxygen molecules. The oxygen sensor films are prepared by dispersing the probe molecules in a suitable polymer support. The polymer matrix for oxygen sensors should have high oxygen permeability and hence silicone polymers are a natural choice. These optical sensors find use not only for biological monitoring of oxygen but also for measuring surface pressure distribution for wind tunnel studies. The oxygen sensitive coating used for surface pressure measurement is known as pressure sensitive paint (PSP) and this specific application is referred as "luminescence barometry" [7-14].

We have been working on the development of PSPs, especially on the paints using pyrene as the pressure sensor and silicone polymer as the binder [15-17]. There are certain advantages for using pyrene as the active luminophore for PSP since it has high pressure sensitivity and low temperature

\footnotetext{
* Corresponding author. Tel.: +91-80-5086251; fax: +91-80-5210113. E-mail address: bharathi@css.cmmacs.ernet.in (B.J. Basu).
}

coefficient at ambient temperature [14,15], but pyrene-based PSPs lack stability and undergo steady degradation during the wind tunnel experiment and even under storage conditions. We had carried out a detailed study of the mechanism of degradation of pyrene-based PSPs and found that the degradation of intensity of the PSP coating was due to evaporation losses of pyrene aided by diffusion of pyrene in silicone polymer [18]. In order to improve the stability of the PSP coatings, it is necessary to arrest the diffusion and evaporation of luminophore molecules from PSP coatings. We have determined the diffusion coefficient of pyrene in silicone polymer coatings prepared with commercially available silicone resins of different viscosities and found that the diffusion of pyrene was independent of the viscosity of the resin [19]. The high diffusion rate of pyrene in silicone coatings is due to the side group rotation and segmental motion of polymer chains in silicone polymers, which create free volume for small molecules to diffuse through. It has been reported that pyrene derivatives like 1-pyrene butyric acid (PBA) have suppressed molecular mobility in silicone films [1]. Hence pressure sensor coatings were prepared by replacing pyrene by pyrene derivatives with lower molecular mobility and their photophysical properties were studied.

Ishiji and Kaneko have studied the photoluminescence of PBA incorporated into silicone film and its potential application as an oxygen sensor [1]. The PBA concentration of 
the silicone films used in their study was low and the emission spectrum of the film exhibited only monomer peaks and no excimer peak. Lubbers and Opitz also have shown that the monomer emission of a layer of PBA stabilized by ethyl cellulose could be used as an oxygen sensor [6]. For PSP applications, the excimer emission of pyrene is utilized because of its higher oxygen quenching, larger Stokes' shift, and higher quantum efficiency than the monomer emission $[14,15]$. When pyrene molecules are embedded in a silicone film or coating, it exhibits highly intense excimer emission with good oxygen quenching sensitivity (OQS). But it is not established whether any of the commercially available pyrene derivative could be used as a substitute for pyrene.

In the present study, photophysical characteristics of commercially available pyrene derivatives were examined with a view to find a suitable luminophore as a substitute for pyrene in the paint. The pyrene derivatives selected for this study were: 1-pyrene methanol (PMO), 1-pyrene butanol (PBO), 1-pyrene butyric acid, and 1-pyrene acetic acid (PAA). A comparison of the oxygen sensor performance of these four pyrene derivatives with that of pyrene in silicone polymer matrices is made and the results are discussed.

\section{Experimental}

\subsection{Materials}

Pyrene and pyrene derivatives (PBO, PMO, PBA, and PAA) were procured from Sigma Aldrich Corporation. The silicone resins, RTV IS 9188, RTV IS 8008, and RTV 11 were obtained from GE Silicones and DMS S42 and methyl triethoxysilane were obtained from ABCR GmbH, Germany. RTV IS 9188 (clear, neutral cure) and RTV IS 8008 (clear, acetoxy cure) are one component silicone resins. Acetoxy cure resins release acetic acid during the curing process and neutral cure resins release alcohol, ammonia or ketoxime depending on the functional group in the resin. RTV 11 is a white, two component condensation cure silicone resin which is cured by the addition of dibutyl tin dilaurate $(0.5 \%)$ as catalyst. DMS S42 is a silanol-terminated PDMS with a molecular weight of 77,000. Methyl triethoxysilane (40\%) as cross linker and dibutyl tin dilaurate $(0.5 \%)$ as catalyst were added to DMS S42 to prepare the silicone coatings. Chloroform and toluene (sulfur free) were used as solvents.

Ten commercially available pyrene derivatives were tested for their oxygen quenching sensitivity in solution and silicone films. Two of the derivatives, 1,3,6,8-pyrene tetrasulfonic acid (PTS) and 8-hydroxy-1,3,6-pyrene trisulfonic acid (HPTS) were not soluble in chloroform or toluene and were soluble in methanol. Silicone coatings of PTS and HPTS were prepared by adsorbing them on silica gel from their methanolic solutions and mixing the dye-adsorbed silica gel with silicone resin. But the coatings of PTS and HPTS were not oxygen sensitive. 1-Pyrene carboxylic acid (PCA) was insoluble in almost all solvents. Solutions and silicone coat- ings of 1-aminopyrene (AP) were highly unstable and decomposed on exposure to UV radiation. In the case of pyrene carboxaldehyde and $N$-(1-pyrenyl) maleimide, the oxygen quenching sensitivity of their coatings was negligibly small. Hence these pyrene derivatives were not suitable for preparing PSP coatings. Other pyrene derivatives like PBO and PMO were soluble in toluene and chloroform. PBA and PAA were sparingly soluble in toluene but a dilute solution could be prepared using chloroform. Four milligram of each derivative was dissolved in $10 \mathrm{ml}$ solvent. The concentrations of solutions of pyrene, PBO, PMO, PBA, and PAA were $2.0 \mathrm{mM}, 1.46 \mathrm{mM}, 1.72 \mathrm{mM}, 1.39 \mathrm{mM}$, and $1.54 \mathrm{mM}$, respectively.

Sensor coatings were prepared by mixing $1 \mathrm{~g}$ of silicone resin with $5 \mathrm{ml}$ of a solution containing $2.0 \mathrm{mg}$ of pyrene or pyrene derivative $(1.0 \mathrm{mg}$ for PAA due to solubility problem). The mixture was sprayed onto $15 \mathrm{~cm} \times 5 \mathrm{~cm}$ aluminum sheets of $0.3 \mathrm{~mm}$ thickness coated with a white reflecting undercoat [15]. This undercoat was prepared by spraying a two-component white paint onto the clean and dry aluminum sheets and curing for $12 \mathrm{~h}$ at room temperature. The sensing layer was allowed to cure for $24 \mathrm{~h}$ at room temperature. Five sensor specimens of $3 \mathrm{~cm} \times 5 \mathrm{~cm}$ were cut from each aluminum sheet sprayed with each mixture. The thickness of the film was about $30-40 \mu \mathrm{m}$.

\subsection{Methods}

\subsubsection{Fluorescence measurements}

Fluorescence emission spectra were recorded using a fiber optic spectrometer, model SD1000 from Ocean Optics, Inc USA and a $300 \mathrm{~W}$ Xenon Arc Lamp (model no. 6258 from Oriel Instruments) as source. The infrared radiation from the xenon lamp was filtered by means of a water filter. A bandpass filter (Oriel cat. no. 51650) was used to transmit UV radiation from the source in the wavelength range of $300-380 \mathrm{~nm}$ and to illuminate the specimens with UV radiation. The specimens were mounted in a sample chamber fabricated in our laboratory so that emission was measured by the front face technique. Purified nitrogen was passed through the sample chamber to record the emission spectra in the absence of oxygen. The fluorescence emission spectra of the coatings were recorded in air and in the presence of nitrogen. The oxygen quenching sensitivity of the specimen was calculated using the equation, $\left(I_{\mathrm{N}_{2}}-I_{\text {air }}\right) \times 100 / I_{\mathrm{N}_{2}}$ where $I_{\mathrm{N}_{2}}$ and $I_{\text {air }}$ are the excimer intensity values of the specimens in nitrogen and air, respectively.

\section{Results and discussion}

\subsection{Photophysical characteristics of pyrene derivatives in solution}

The absorbance spectra of the solutions of pyrene and pyrene derivatives in chloroform were recorded. The 


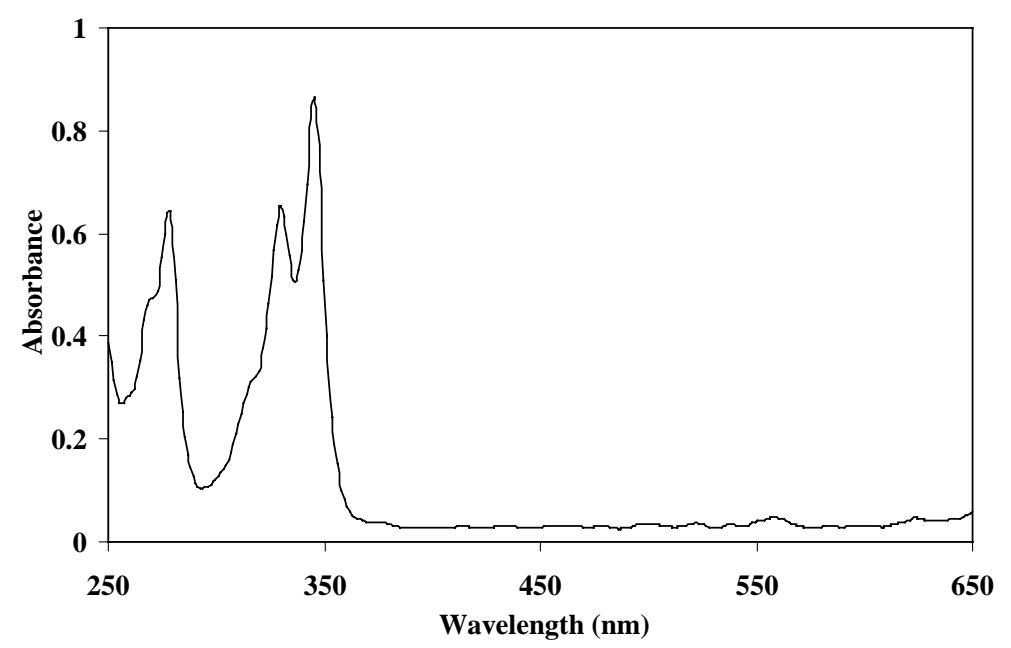

Fig. 1. Absorbance spectrum of a solution of $\mathrm{PBO}$ in toluene. Concentration of $\mathrm{PBO}=0.02 \mathrm{mM}$.

concentration of luminophore in solution was $0.02 \mathrm{mM}$. It was found that all the pyrene derivatives absorbed in the UV region. The absorbance peaks of PBO, PMO, PBA, and PAA were similar to that of pyrene and there was only a slight shift to longer wavelength by about $8-10 \mathrm{~nm}$. The absorbance spectrum of PBO is shown in Fig. 1. The wavelength of maximum absorbance was about 345$346 \mathrm{~nm}$.

The fluorescence emission spectra of pyrene and pyrene derivatives in solution were recorded. The solutions were de-aerated by bubbling nitrogen through the solution in the quartz cuvette for about 4-5 min and the emission spectra of the de-aerated solutions were recorded. The fluorescence emission spectra of a $2.0 \mathrm{mM}$ solution of pyrene in toluene before and after de-aeration are shown in Fig. 2. There were three prominent emission peaks for pyrene. The sharp peaks at 393.1 and $417.3 \mathrm{~nm}$ were due to monomer emission and the broad peak at $477.0 \mathrm{~nm}$ was due to pyrene excimer emis- sion. All the emission peaks of pyrene were found to be oxygen sensitive. The OQS of the excimer emission was found to be $92.5 \%$.

The fluorescence emission spectra of solutions of $\mathrm{PBO}$ and $\mathrm{PMO}$ in toluene and chloroform, respectively before and after de-aeration are shown in Fig. 3. The emission spectrum of a solution of PBO $(1.46 \mathrm{mM})$ in toluene consisted of three peaks, at $396.6 \mathrm{~m}, 419.6$, and $478.0 \mathrm{~nm}$ (Fig. 3A). All the emission peaks of $\mathrm{PBO}$ were oxygen sensitive. The emission spectrum of PMO solution $(1.72 \mathrm{mM})$ also consisted mainly of three peaks as shown in Fig. 3B. The excimer peaks of PBO and PMO solutions were more oxygen sensitive than their monomer peaks. The fluorescence emission spectra of solutions of PBA and PAA in chloroform before and after de-aeration are shown in Fig. 4. There were three prominent emission peaks for PBA at about 399.0, 423.0, and $479.0 \mathrm{~nm}$ (Fig. 4A). Peaks similar to that of PBA were observed in the case of PAA as shown in Fig. 4B. The excimer peak of PAA

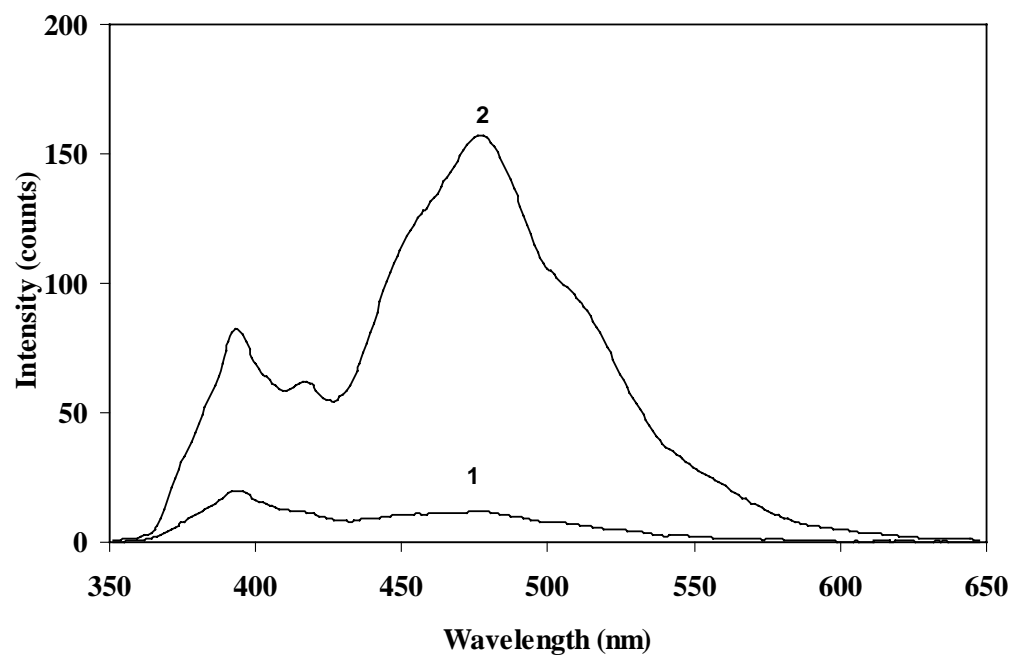

Fig. 2. Fluorescence emission spectra of a solution of pyrene in toluene; (1) before deaeration and (2) after deaeration. Concentration of pyrene $=2.0 \mathrm{mM}$. 

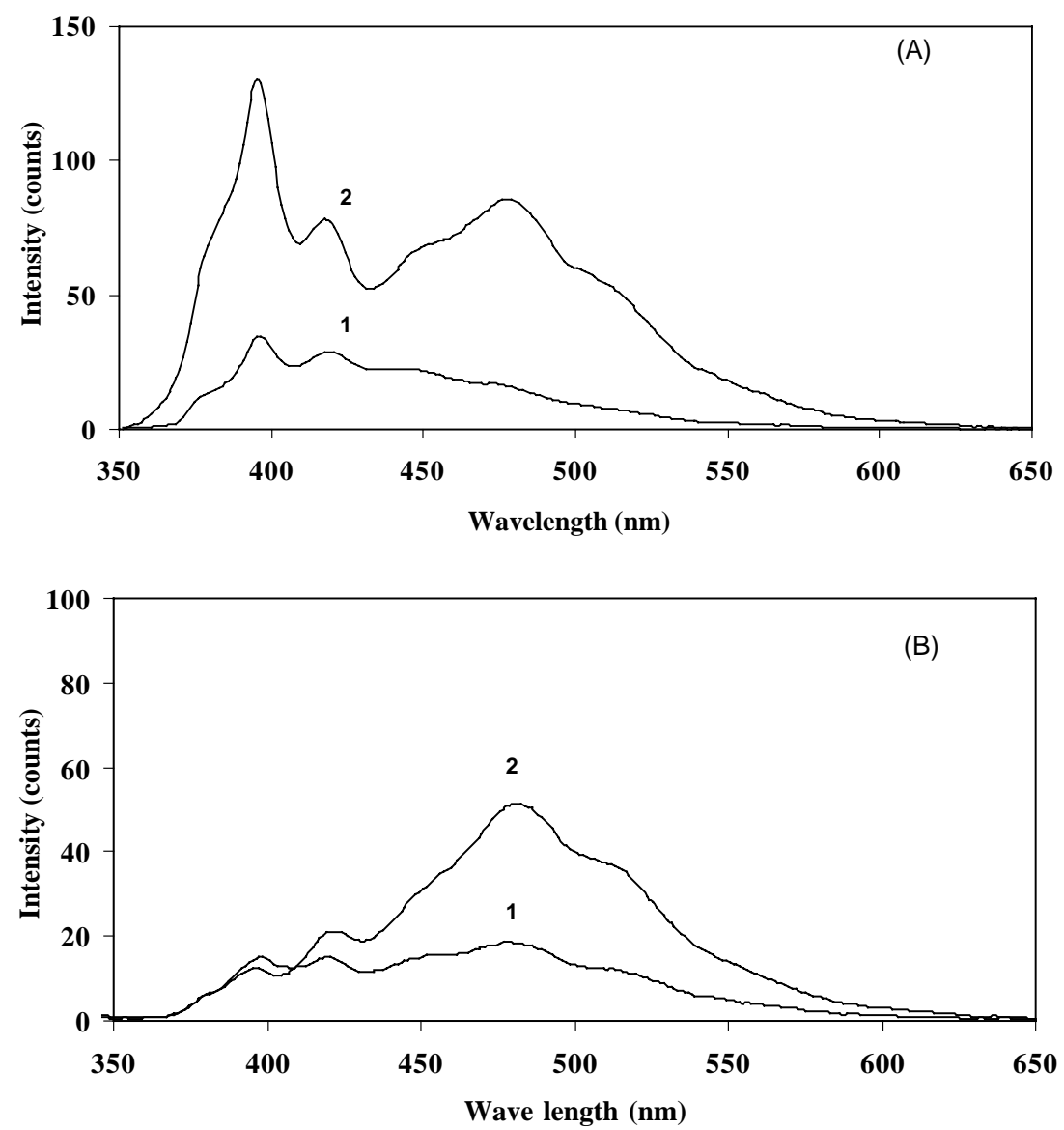

Fig. 3. Fluorescence emission spectra of solutions of (A) PBO $(1.46 \mathrm{mM})$ in toluene; and (B) PMO (1.72 $\mathrm{mM})$ in chloroform (1) before deaeration and (2) after deaeration.

was less sensitive to oxygen quenching compared to that of PBA. Typical fluorescent intensity values (for monomer and excimer emissions) and oxygen quenching sensitivity of the solutions of pyrene and pyrene derivatives are shown in Table 1. It was found that excimer emission was more oxygen sensitive than monomer emissions for pyrene and pyrene derivatives in solution.

\subsection{Fluorescence emission of the silicone coatings of pyrene derivatives}

Coatings of pyrene and pyrene derivatives with a two-component condensation cure silicone resin (GE RTV 11) were prepared as described in Section 2.1. The concentrations of pyrene, PBO, PMO, PBA, and PAA in the coatings were 10.0, 7.3, 8.6, 6.9, and $3.8 \mathrm{mM}$, respectively. The fluorescence emission spectra of these coatings were recorded in air and in the presence of nitrogen and these are shown in Figs. 5-7.

The fluorescence emission spectra of a coating of pyrene in air and in the presence of nitrogen are shown in Fig. 5. It was found that the excimer emission of pyrene in the coating was enhanced about five-fold in the presence of nitrogen. The OQS for the excimer emission of pyrene in sili- cone coating was about $80-81 \%$. Fig. 6 shows the fluorescence emission spectra of coatings of PBO and PMO in air and in the presence of nitrogen. The spectra of PBO coating showed three monomer peaks (at 378, 398, and $418 \mathrm{~nm}$ ) and

Table 1

Photophysical characteristics of pyrene derivatives in solution

\begin{tabular}{lllll}
\hline Luminophore & $\begin{array}{l}\lambda_{\max } \\
(\mathrm{nm}) \text { of } \\
\text { emission }\end{array}$ & \multicolumn{2}{l}{$\begin{array}{l}\text { Fluorescent emission } \\
\text { intensity (counts) }\end{array}$} & $\begin{array}{l}\text { Oxygen } \\
\text { quenching } \\
\text { sensitivity (\%) }\end{array}$ \\
\cline { 3 - 4 } & & $\begin{array}{l}\text { Before } \\
\text { de-aeration }\end{array}$ & $\begin{array}{l}\text { After } \\
\text { de-aeration }\end{array}$ \\
\hline Pyrene & 393.1 & 19.9 & 82.4 & 75.9 \\
& 417.3 & 11.0 & 60.2 & 83.4 \\
& 477.0 & 11.8 & 156.9 & 92.5 \\
PBO & 396.6 & 34.7 & 105.4 & 67.1 \\
& 419.6 & 28.9 & 80.1 & 63.9 \\
& 478.0 & 16.0 & 90.9 & 82.4 \\
PMO & 421.0 & 14.8 & 21.2 & 30.2 \\
& 480.0 & 18.3 & 51.1 & 64.2 \\
PBA & 399.0 & 24.8 & 31.9 & 22.4 \\
& 422.0 & 19.3 & 33.5 & 42.4 \\
& 479.0 & 15.1 & 51.1 & 70.5 \\
PAA & 479.0 & 12.6 & 25.2 & 50.0 \\
\hline
\end{tabular}



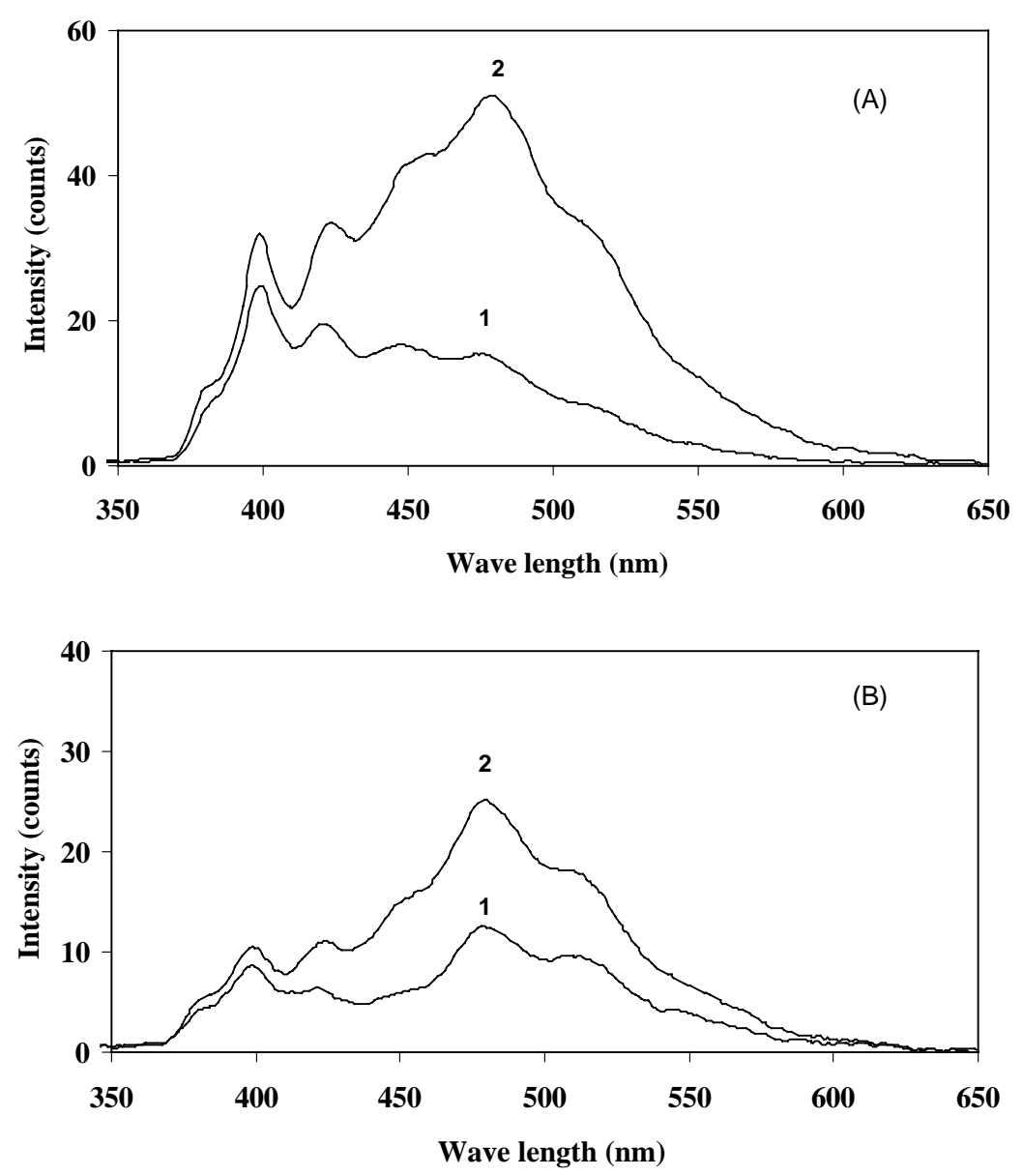

Fig. 4. Fluorescence emission spectra of solutions of (A) PBA (1.39 mM) in chloroform; and (B) PAA (1.54 mM) in chloroform (1) before deaeration and (2) after deaeration.

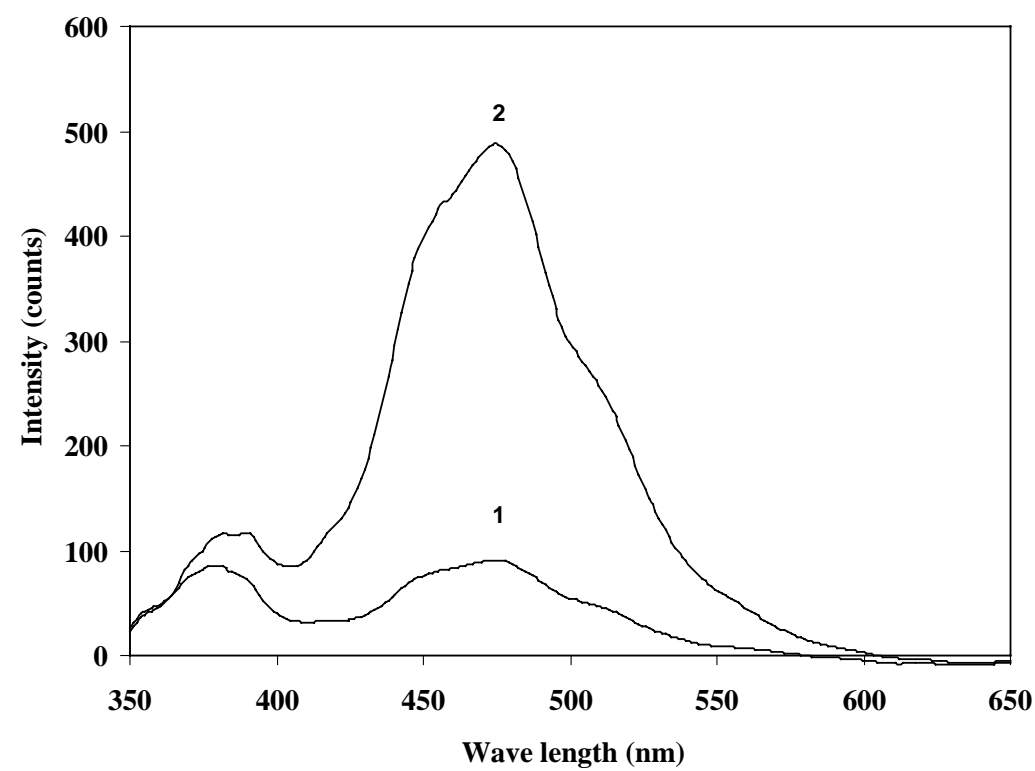

Fig. 5. Fluorescence emission spectra of a coating of pyrene prepared with the silicone resin RTV 11, (1) in air and (2) in the presence of nitrogen. 

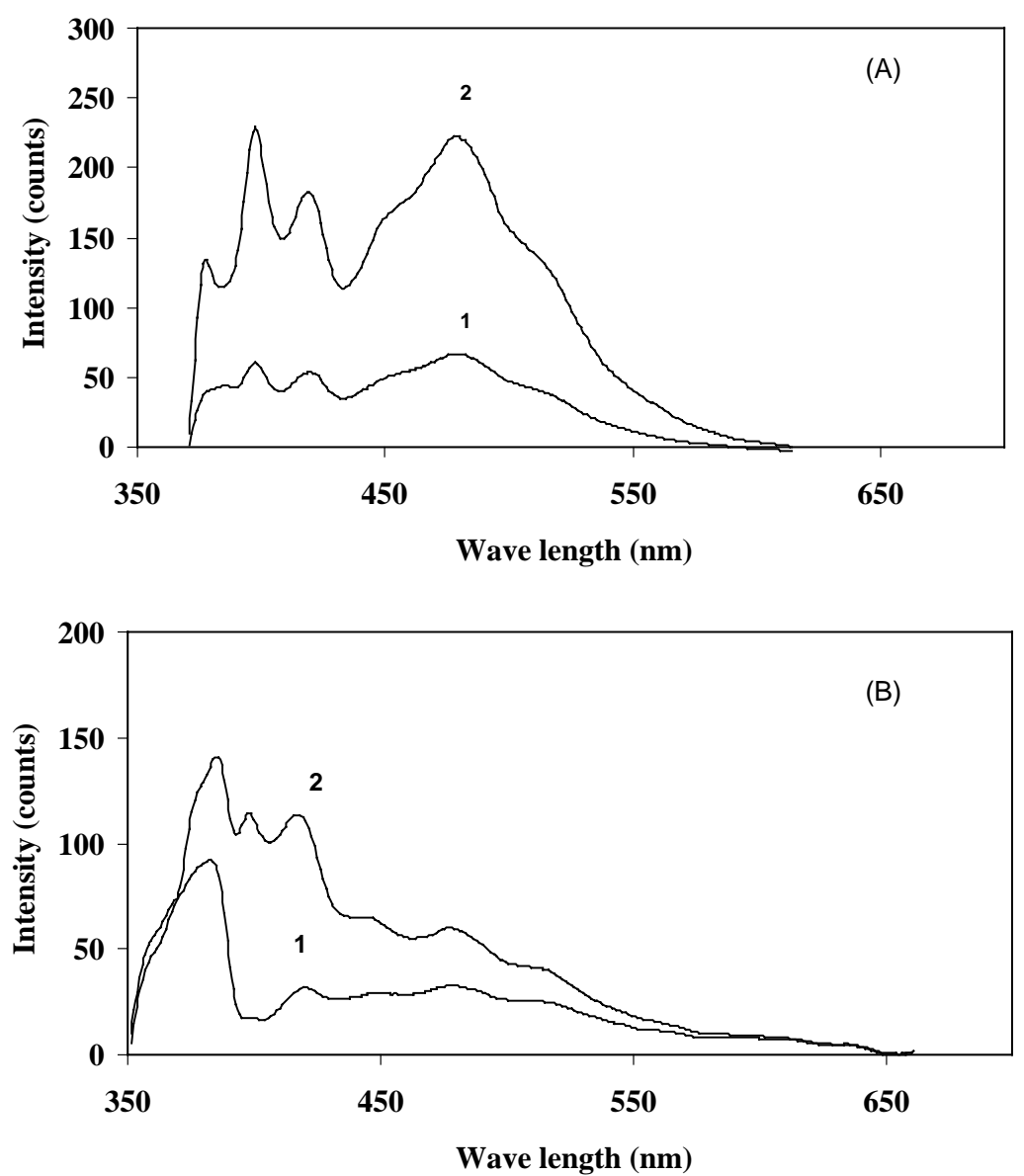

Fig. 6. Fluorescence emission spectra of coatings of (A) PBO; and (B) PMO prepared with RTV 11, (1) in air and (2) in the presence of nitrogen.

an excimer peak at $479 \mathrm{~nm}$. The OQS values for monomer and excimer emissions of PBO coating were about $70 \%$. It was found that the monomer emissions of PMO were more oxygen sensitive than the excimer emission as shown in Fig. 6B. Fig. 7 shows the fluorescence emission spectra of the coatings of PBA and PAA in air and in the presence of nitrogen. The excimer emission of PBA was less intense and the oxygen quenching of excimer emission was poor. The monomer emission of PBA at $418 \mathrm{~nm}$ was more oxygen sensitive than the excimer emission. The photophysical characteristics of PAA were similar to that of PBA and it had lower OQS for excimer emission. The fluorescence intensity values and OQS values of the coatings of pyrene and pyrene derivatives are shown in Table 2.

It can be seen from Table 2 that pyrene coating has shown the highest OQS for its excimer emission. This can be attributed to the high mobility of pyrene molecules in silicone polymer matrix and also to the longer lifetime of pyrene excimer. Among the coatings of pyrene derivatives, OQS for excimer emission increased in the order $\mathrm{PBO}>\mathrm{PMO}>$ $\mathrm{PBA} \geq \mathrm{PAA}$. Both PBA and PAA coatings had poor OQS for excimer emission. The monomer emissions of PMO, PBA, and PAA in silicone polymer were more oxygen sensitive than their excimer emissions.

\subsection{The effect of binder on the oxygen quenching of the sensor coating}

It is known that binder has an important role in deciding the oxygen sensor performance [20]. Commercially

Table 2

Photophysical characteristics of pyrene derivatives in silicone coatings with RTV11

\begin{tabular}{lllll}
\hline Luminophore & $\begin{array}{l}\lambda_{\mathrm{em}} \\
(\mathrm{nm})\end{array}$ & \multicolumn{2}{l}{$\begin{array}{l}\text { Fluorescent emission } \\
\text { intensity in }\end{array}$} & $\begin{array}{l}\text { Oxygen } \\
\text { quenching } \\
\text { sensitivity (\%) }\end{array}$ \\
\cline { 3 - 4 } & & Air $\left(I_{\text {air }}\right)$ & Nitrogen $\left(I_{\mathrm{N}_{2}}\right)$ & \\
\hline Pyrene & 381.5 & 84.8 & 116.9 & 27.4 \\
& 389.6 & 72.8 & 116.8 & 37.7 \\
& 475 & 90.8 & 478.4 & 81.0 \\
PBO & 397.5 & 61.0 & 230.1 & 73.5 \\
& 418.3 & 53.2 & 182.3 & 70.8 \\
& 479.1 & 67.0 & 222.5 & 69.9 \\
PMO & 417.2 & 30.3 & 113.5 & 73.3 \\
& 479.1 & 32.9 & 59.8 & 45.0 \\
PBA & 418.3 & 16.7 & 52.3 & 68.0 \\
& 476 & 28.0 & 30.8 & 9.1 \\
PAA & 418.3 & 31.0 & 90.0 & 65.6 \\
& 476 & 36.1 & 39.5 & 8.6 \\
\hline
\end{tabular}



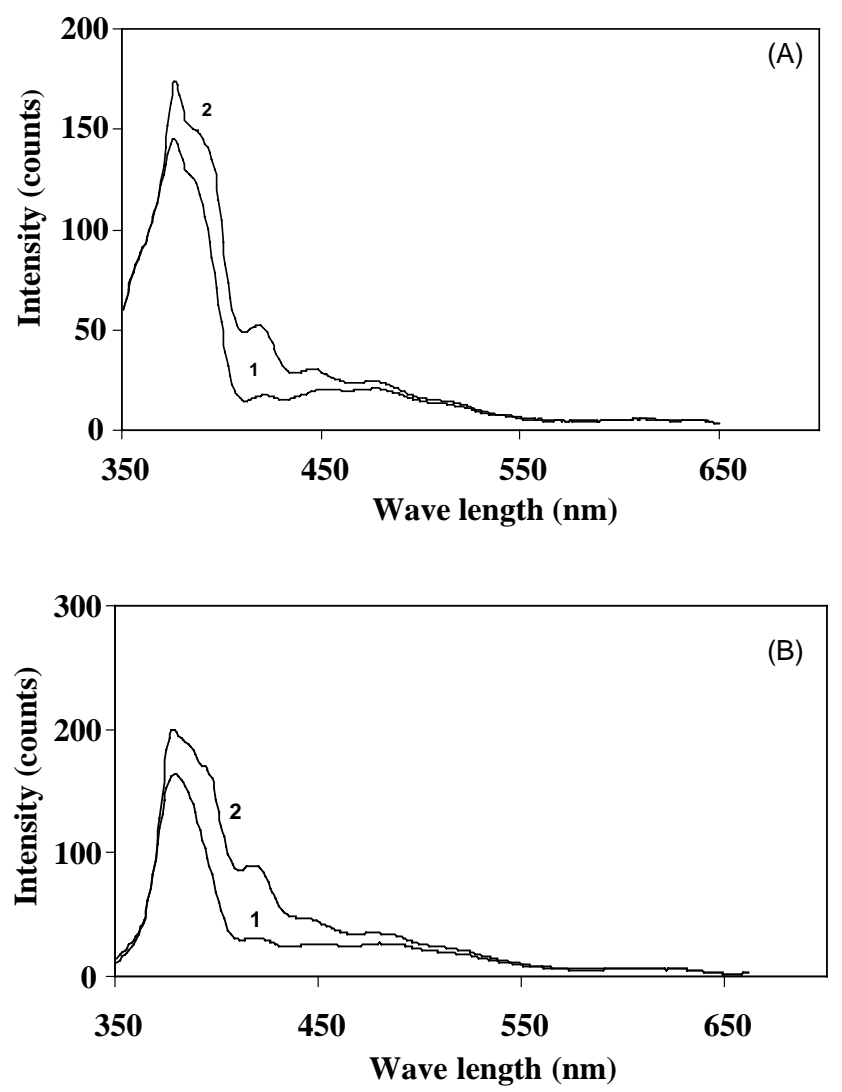

Fig. 7. Fluorescence emission spectra of coatings of (A) PBA; and (B) PAA prepared with RTV 11, (1) in air and (2) in the presence of nitrogen.

available silicone prepolymers contain solvents, fillers, low molecular weight crosslinkers, catalysts, and other additives. These unknown components may affect the oxygen quenching of the sensor molecules. Therefore, the effect of different silicone resins as binders on the oxygen quenching of sensor coatings was studied. Three silicone polymers, RTV IS 9188, RTV IS 8008, and DMS S42 were selected for this study.

Coatings of pyrene and pyrene derivatives with these silicone resins were prepared in a similar manner as described in Section 2.1. The concentration of the luminophore in the coating was the same as for the coatings with RTV 11 . The fluorescence emission spectra of the coatings were recorded in air and in the presence of nitrogen. Since excimer emission of pyrene is useful for PSP measurements, the intensity values of the excimer emission of these coatings were measured and the OQS was calculated and these are shown in Table 3.

It was found that the oxygen quenching of the excimer emission of pyrene derivatives in different silicone coatings was lower than that of pyrene. The OQS of excimer emission of coatings of pyrene with all resins was high and was about $80-81 \%$. Coatings of PBO with RTV 11 exhibited an OQS of $69-70 \%$ for the excimer emission whereas the OQS was lower for coatings of other pyrene derivatives. The oxygen quenching of excimer emission
Table 3

Oxygen quenching sensitivity of the excimer emission of coatings of pyrene and pyrene derivatives with two silicone resins

\begin{tabular}{lcccc}
\hline Luminophore & \multicolumn{5}{l}{$\begin{array}{l}\text { Oxygen quenching sensitivity (\%) of the } \\
\text { excimer emission of sensor coatings with }\end{array}$} \\
\cline { 2 - 5 } & RTV 11 & RTV IS 9188 & RTV IS 8008 & DMS S42 \\
\hline Pyrene & 81.0 & 79.8 & 79.9 & 80.4 \\
PBO & 69.5 & 44.4 & 58.2 & 67.1 \\
PMO & 45.0 & 35.1 & 31.0 & 42.7 \\
PBA & 9.5 & 9.1 & 8.5 & 9.1 \\
PAA & 8.6 & 7.6 & 7.5 & 8.4 \\
\hline
\end{tabular}

was poor $(<10 \%)$ for coatings of PBA and PAA in all resins.

The OQS of the excimer emission of PBO coating was found to depend on the type of silicone resin used. Coatings of PBO with RTV 11 exhibited a higher OQS for the excimer emission than coatings of PBO with other resins like IS 9188 and IS 8008 which are single component resins with neutral cure and acetoxy cure, respectively. The OQS of PBO coatings with DMS S42 and RTV 11 were comparable. DMS S42 is a silicone resin with known molecular structure (hydroxy terminated PDMS with a molecular weight of 77,000) and methyl triethoxysilane was used as crosslinker to prepare the coating. Therefore, the coating with DMS S42 will have long chains of PDMS with lesser cross linking since the functional groups are at the end of the chain. It has been reported that coatings with higher crosslinking exhibit lower oxygen quenching [20]. This may be due to the decreased oxygen permeability of silicone polymers with higher crosslinking. In the case of the other three commercial silicone resins, the composition is unknown. But our results indicate that the coatings of IS 9188 and IS 8008 may have higher crosslinking due to branched chains in their molecular structure. Since RTV 11 is a condensation cure resin, it is most likely a hydroxy-terminated PDMS resin and may have higher oxygen permeability than IS 9188 and IS 8008 .

\subsection{Oxygen quenching kinetics of the luminophores dissolved in solvents and polymer matrices}

It is possible to explain these results on the basis of the oxygen quenching kinetics [21]. Dynamic quenching or diffusion-controlled quenching is described by the Stern-Volmer equation

$\frac{I_{0}}{I}=\frac{\tau_{0}}{\tau}=1+k_{\mathrm{q}} \tau_{0}\left[\mathrm{O}_{2}\right]$

where $I$ and $I_{0}$ are the intensity, $\tau$ and $\tau_{0}$ are the lifetimes of the luminophore at a molar concentration of oxygen, $\left[\mathrm{O}_{2}\right]$ and in the absence of oxygen respectively, and $k_{\mathrm{q}}$ is the quenching rate constant.

According to Henry's law, the equilibrium oxygen concentration in the polymer coating is proportional to the oxygen partial pressure, $p \mathrm{O}_{2}$ in contact with the surface of 
the coating under ambient pressure and temperature conditions

$\left[\mathrm{O}_{2}\right]=s \mathrm{O}_{2} p \mathrm{O}_{2}$

where $s \mathrm{O}_{2}$ is the solubility of oxygen in the coating.

For diffusion controlled quenching, the quenching rate constant, $k_{\mathrm{q}}$ is related to the diffusion rate constant, $k_{\mathrm{d}}$ for the collision between oxygen and the excited luminophore molecules by the equation

$k_{\mathrm{q}}=\alpha k_{\mathrm{d}}$

where $\alpha$ is the probability that a collision leads to quenching and is assumed to be equal to unity in many cases, especially for the quenching of singlet excited states. According to Smoluchowski equation relating the diffusion rate constant and the diffusion coefficient

$k_{\mathrm{d}}=\frac{4 \pi N \sigma D}{1000}$

where $N$ is the Avogadro's number and $\sigma$ is the collision radius of the oxygen-dye complex. But since the diffusion coefficient of oxygen in the polymer $\left(D_{\mathrm{O}_{2}}\right)$ is usually much higher than the diffusion coefficient of the luminophore dye $\left(D_{\mathrm{L}}\right)$

$D=D_{\mathrm{O}_{2}}+D_{\mathrm{L}} \approx D_{\mathrm{O}_{2}}$

Therefore, the Eq. (4) becomes

$k_{\mathrm{d}}=\frac{4 \pi N \sigma D_{\mathrm{O}_{2}}}{1000}$

Substitution of Eqs. (2), (3) and (6) in Eq. (1) gives

$\frac{I_{0}}{I}=\frac{\tau_{0}}{\tau}=\frac{1+4 \pi N \sigma \alpha \tau_{0}\left(D_{\mathrm{O}_{2}} \times s \mathrm{O}_{2}\right) p \mathrm{O}_{2}}{1000}$

The product of $D_{\mathrm{O}_{2}}$ and $s \mathrm{O}_{2}$ gives the oxygen permeability of the polymer matrix $(P)$. It is evident from Eq. (7) that the oxygen quenching sensitivity of a sensor film or coating depends on the oxygen permeability and unquenched luminescence lifetime, $\tau_{0}$. Since silicone-based polymers have high oxygen permeability, they are often used as the matrix for oxygen sensors. In order to get high oxygen sensitivity, one has to select a luminophore with longer unquenched lifetime (preferrably $>100 \mathrm{~ns}$ ).

The lifetimes of monomer emission of pyrene and PMO in various solvents and polymer matrices like polystyrene (PS), polymethyl methacrylate (PMMA) and polyvinyl chloride (PVC) have been reported earlier [22]. These authors have found that the decay of fluorescence emission in the monomeric region of pyrene and PMO is monoexponential in solvents and polymer matrices. The longest lifetime, $414 \mathrm{~ns}$ was exhibited by pyrene in deaerated cyclohexane and lifetimes of pyrene in more polar solvents were shorter. The lifetimes of PMO were shorter than the lifetime of pyrene in all solvents and polymer matrices. Solutions of pyrene and PMO in chloroform have an unquenched lifetime of 133 and $101 \mathrm{~ns}$, respectively [22]. The monomer emission of a solution of PBA in toluene showed a single exponential decay with a lifetime of $61.5 \mathrm{~ns}$ [1]. Ishiji and Kaneko have studied the monomer emission decay of PBA in silicone film under argon atmosphere and found that the emission exhibits multicomponent decay which fitted well by double exponential analysis with two lifetime values [1]. They have reported that the monomer lifetimes of PBA in silicone were longer than the value in solution. This explains the higher OQS (68\%) obtained for monomer emission of PBA at $418 \mathrm{~nm}$ in silicone coating (Table 2). The multicomponent emission decay and longer lifetime of PBA in silicone film indicate that the molecular mobility of PBA in film is suppressed. Because of suppressed molecular mobility of PBA in silicone film, excimers are not formed. At higher PBA concentrations in the film, ground state dimers may be formed which give rise to an excimer peak with low intensity. On the other hand, silicone films containing pyrene give rise to excimer peaks with high intensity even at low concentrations due to the high molecular mobility of pyrene molecules in silicone polymer matrix. The lifetimes of excimer emissions of pyrene and pyrene derivatives in silicone polymer matrix are not available in literature.

The excimer fluorescence decay characteristics of coatings of pyrene and PBA prepared with silicone resin, IS 9188 under ambient conditions was studied. It was found that the excimer fluorescence decay of pyrene could be fitted by a bi-exponential equation. The profile comprised a growth followed by a decay with lifetimes, $\tau_{1}=10.64 \mathrm{~ns}$ (growth) and $\tau_{2}=30.24 \mathrm{~ns}$ (decay). From Eq. (1), $I_{0} / I=$ $\tau_{0} / \tau$. The intensity in the presence of nitrogen, $I_{\mathrm{N}_{2}}$ is nearly equal to $I_{0}$, the intensity in the absence of oxygen. Therefore, $I_{0} / I \approx I_{\mathrm{N}_{2}} / I_{\text {air }} \approx \tau_{0} / \tau$ (air). It can be seen from Table 2 that $I_{\mathrm{N}_{2}} / I_{\text {air }}=5.27$ at $475 \mathrm{~nm}$ for a pyrene coating. Hence, $\tau_{0}=30.24 \times 5.27=159.3 \mathrm{~ns}$ for pyrene in silicone polymer matrix. The fluorescence decay profile of a coating of PBA in IS 9188 in the presence of air did not show a growth time but the decay was bi-exponential with lifetimes, $\tau_{1}=28.97 \mathrm{~ns}(71.60 \%)$ and $\tau_{2}=11.01 \mathrm{~ns}(28.40 \%)$. From Table $2, I_{\mathrm{N}_{2}} / I_{\text {air }}=1.15$ at $476 \mathrm{~nm}$ for a PBA coating. Hence the unquenched lifetime, $\tau_{0}=28.97 \times 1.15=33.32 \mathrm{~ns}$ for PBA. Thus, it was seen that $\tau_{0}$ is low for silicone coating of PBA $(<100 \mathrm{~ns})$ and hence it has poor OQS. The lifetimes should fall within the time scale of diffusion-controlled bimolecular reaction so that the fluorescent molecules are quenched dynamically.

\section{Conclusions}

This study has shown that the photophysical characteristics of pyrene derivatives in solution and in the coating differed from that of pyrene. The excimer emission was more oxygen sensitive than monomer emissions for pyrene and pyrene derivatives in solution. Silicone coatings of pyrene exhibited high oxygen quenching for the excimer emission. But when pyrene derivatives were dispersed in silicone polymer matrix, their photophysical characteristics changed 
drastically. Among the coatings of pyrene derivatives, OQS for excimer emission increased in the order $\mathrm{PBO}>\mathrm{PMO}>$ $\mathrm{PBA} \geq \mathrm{PAA}$. The OQS of excimer peak and monomer peaks were comparable for $\mathrm{PBO}$ coating whereas the oxygen quenching of excimer emission of PMO, PBA, and PAA was lower than the OQS of their monomer emissions. The coatings of PBA and PAA had poor OQS $(<10 \%)$ for excimer emission. It was found that OQS of the excimer emission of PBO coating depended on the type of silicone resin used. The coatings of PBO with the two-component silicone resin RTV 11 and silanol-terminated PDMS resin were found to have higher OQS than coatings of PBO with single component resins. Therefore, PBO may find use as a potential luminophore along with silanol-terminated PDMS resin as binder for PSP applications provided it fulfils other PSP requirements.

\section{Acknowledgements}

The authors would like to express their gratitude to Dr. B.R. Pai, Director, NAL for his support of this work. We are grateful to Dr. A.K. Mishra, Department of Chemistry, IIT, Madras for providing the fluorescence decay profiles of two samples. We thank Dr. C. Anandan for his help in modifying the experimental setup for fluorescence measurements. Our sincere thanks are also due to Mr. A. Thirumurugan, Mr. A.R. Dinesh and Mr. N. Senthil Kumar for measurements of fluorescence intensity of the coatings.

\section{References}

[1] T. Ishiji, M. Kaneko, Photoluminescence of pyrenebutyric acid incorporated into silicone film as a technique in luminescent oxygen sensing, Analyst 120 (1995) 1633-1638.

[2] A. Sharma, O.S. Wolfbeis, Fiberoptic oxygen sensor based on fluorescence quenching and energy transfer, Appl. Spectrosc. 42 (1988) 1009-1011.

[3] E.D. Lee, T.C. Werner, R. Seitz, Luminescence ratio indicators for oxygen, Anal. Chem. 59 (1987) 279-283.

[4] H.W. Kronies, H.J. Marsoner, A fluorescence-based sterilizable oxygen probe for use bioreactors, Sens. Actuators 4 (1983) 587592.

[5] W. Xu, R. Schmidt, M. Whaley, J.N. Demas, B.A. Degraff, E.K. Karikari, B.L. Farmer, Oxygen sensors based on luminescence quenching: interactions of pyrene with polymer supports, Anal. Chem. 67 (1995) 3172-3180.

[6] D.W. Lubbers, N. Opitz, Optical fluorescence sensors for continuous measurement of chemical concentrations in biological systems, Sens. Actuators 4 (1983) 641-654.

[7] M. Gouterman, Oxygen quenching of luminescence of pressure sensitive paint for wind tunnel research, J. Chem. Educ. 74 (1997) 697-702.
[8] J. Kavandi, J. Callis, M. Gouterman, G. Khalil, D. Wright, E. Green, Luminescent barometry in wind tunnels, Rev. Sci. Instrum. 61 (1990) 3340-3347.

[9] B.G. McLachlan, J.L. Kavandi, J.B. Callis, M. Gouterman, E. Green, G. Khalil, D. Barns, Surface pressure field mapping using luminescent coatings, Exp. Fluids 14 (1993) 33-41.

[10] J.H. Bell, E.T. Schairer, L.A. Hand, R.D. Mehta, Surface pressure measurements using luminescent coatings, Annu. Rev. Fluid Mech. 33 (2001) 155-206.

[11] R.C. Crites, Measurement Techniques, Lecture Series 1995-01, Von Karman Institute for Fluid Dynamics.

[12] T. Liu, B.T. Campbell, S.P. Burns, J.P. Sullivan, Temperature and pressure sensitive luminescent paints in aerodynamics, Appl. Mech. Rev. 50 (1997) 227.

[13] R.H. Engler, DLR PSP system intensity and lifetime measurements, ICIASF'97 Record, IEEE Publication 97CH 36121, pp. 46-56.

[14] Y. Mebarki, Pressure sensitive paints: application in wind tunnels, ONERA NT 1998-6, 1998, pp. 1-143.

[15] B.J. Basu, R. Kannan, I. Rajagopal, K.S. Rajam, Development and evaluation of the first binary pressure sensitive paint NAL-G, NAL Project Document SE 0104 (2001) 1-21.

[16] B.J. Basu, C. Anandan, K.S. Rajam, Investigations on the development of improved binary PSP, NAL G1, NAL Project Document SE 0112 (2001) 1-17.

[17] B.J. Basu, C. Anandan, K.S. Rajam, Development and evaluation of improved binary PSP, NAL G2, NAL Project Document SE 0113 (2001) 1-14.

[18] B.J. Basu, C. Anandan, K.S. Rajam, Study of the mechanism of degradation of pyrene-based pressure sensitive paints, Sens. Actuators B 94 (2003) 257-266.

[19] C. Anandan, B.J. Basu, K.S. Rajam, Investigations of the effect of viscosity of rein on the diffusion of pyrene in silicone polymer matrix using steady state fluorescence technique, Eur. Polym. J. 40 (2004) $335-342$.

[20] H. He, R.J. Fraatz, M.J.P. Leiner, M.M. Rehn, J.K. Tusa, Selection of silicone polymer matrix for optical gas sensing, Sens. Actuators B 29 (1995) 246-250.

[21] X. Lu, M.A. Winnik, Luminescence quenching in polymer/filler nanocomposite films used in oxygen sensors, Chem. Mater. 13 (2001) 3449-3463.

[22] P. Hrdlovic, I. Lukac, Monosubstituted derivatives of pyrene Comparison of their spectral behaviour in solution and in polymer matrices, J. Photochem. Photobiol. A: Chem. 133 (2000) 73-82.

\section{Biographies}

Bharathibai J. Basu received her masters degree in chemistry from Calicut University, Kerala, India in 1973 and $\mathrm{PhD}$ from Indian Institute of Science, Bangalore, India in 1995. She started her research career in National Aerospace Laboratories, Bangalore, India in 1974. Research areas of her interest are trace element analysis, electroanalytical chemistry, and spectroscopy. Her current interest is in the development of pressure sensitive paints for wind tunnel studies.

K.S. Rajam is currently heading the Surface Engineering Division, National Aerospace Laboratories, Bangalore, India. She holds a doctorate from Bangalore University. Her technical interests include developing surface modification technologies for aerospace and other engineering applications. She is currently engaged in electroplating, electroless plating, multilayer coatings, electroforming, and pressure sensitive paints. 